\title{
Comportement non drainé de sable Hostun RF très lâche en consolidation anisotrope
}

\section{R. MATIOTTI}

Politecnico di Milano, Dipartimento di Ingegneria Strutturale

Piazza Leonardo da Vinci. 32 20133 Milano, Italie

\section{E. IBRAIM, T. DOANH}

Ecole nationale des Travaux publics de l'État,

Laboratoire Géomatériaux URA 1652

69518 Vaulx-en-Velin

France
Dans cet article, sont présentés les résultats de trois séries d'essais triaxiaux non drainés sur des échantillons de sable d'Hostun RF très lâche, en consolidation isotrope et anisotrope, en compression et en extension. Ils sont ensuite analysés pour vérifier la validité du concept de ligne d'instabilité, introduite par Lade, sur le comportement non drainé en extension et dans des conditions de consolidation anisotrope. L'étude expérimentale confirme que, quelles que soient l'histoire de consolidation et les conditions de cisaillement non drainé, en compression comme en extension, les pics (maximums et minimums) du déviateur de contrainte se trouvent alignés formant une ligne d'instabilité. Celle-ci dépend fortement de l'histoire de consolidation. Une forte anisotropie de consolidation en modifie sensiblement la pente. Le niveau de contrainte au pic augmente avec l'augmentation du coefficient de consolidation anisotrope $\left(\mathrm{K}=\sigma_{3} / \sigma_{1}\right)$. Ce niveau normalisé par rapport au niveau de consolidation suit un modèle exponentiel décroissant. Selon ce modèle, il existe toujours une résistance non drainée à mobiliser en compression comme en extension quelle que soit I'histoire de consolidation, isotrope ou anisotrope.

\section{Undrained behaviour of very loose Hostun RF sand under anisotropic consolidation}

This paper deals with the undrained compression and extension triaxial test results on very loose Hostun RF sand specimens with isotropic and anisotropic consolidation. The tests were performed to verify the applicability of the instability concept of Lade on extension tests of anisotropic specimens. The experimental resuits show that, for the compression and extension test with anisotropic conditions, the stress difference peaks (maxima and minima) can be fitted by a straight line. This instability line depends heavily on consolidation's history. A high anisotropic consolidation modifies the slope of the instability line. The higher anisotropic consolidation coefficients $\left(\mathrm{K}=\sigma_{2} / \sigma_{1}\right)$, the higher the stress peak level. The stress peak level normalized by the stress consolidation level show a decreasing exponential model. For every stress consolidation level there is a peak, even near to the drained sand resistance. 


\section{1}

\section{Introduction}

La liquéfaction statique a été définie comme l'annulation de la contrainte moyenne effective lors d'un essai monotone non drainé par une augmentation continue de la pression interstitielle. Ce phénomène d'instabilité apparaît lors de l'écrasement de l'échantillon de sable très lâche.

Casagrande (1975), en étudiant le comportement du sable, introduit le concept d'état critique, qui correspond à l'indice des vides d'un échantillon de sable atteint en très grandes déformations, lors d'un essai de cisaillement drainé. En plus, il a postulé que le comportement non drainé à l'état critique correspond à celui d'une structure d'écoulernent. La relation entre la contrainte radiale effective à l'état permanent et l'indice des vides génère, dans le plan e-log $\sigma^{\prime}$, une courbe unique F. Pendant un essai de cisaillement drainé, un échantillon avec un indice des vides initial moins grand que celui de l'état critique, dilate, tandis qu'il se contracte lorsque l'indice des vides initial est plus grand. Si l'état du sable se situe dans le domaine contractant lors d'un essai non drainé, sa pression interstitielle augmente et l'échantillon peut atteindre la liquéfaction.

Castro (1969) donnant une série de résultats d'essais triaxiaux non drainés, introduit le concept d'état permanent de déformation: c'est l'état résiduel qui suit le pic de résistance, dans lequel le sable continue à se déformer à volume, état de contrainte et pression interstitielle constants.

Poulos (1981) observe que l'état permanent de déformation est aussi caractérisé par une vitesse de déformation constante.

Sladen et al. (1985) introduisent le concept de surface d'effondrement, définie dans l'espace p'-q-e. Pour des échantillons ayant le même indice des vides initial et avec différentes pressions initiales de consolidation, la ligne reliant les points correspondant au pic du déviateur dans le plan p'-q, pour des chemins triaxiaux non drainés, est une droite qui passe par le point de l'état permanent. Cet état permanent est unique pour un indice des vides donné. Pour diffêrents indices des vides initiaux, des droites parallèles vont générer une surface dans l'espace tridimensionnelle des contraintes-l'indice des vides.

Des échantillons de sable avec une contrainte proche ou sur la surface d'effondrement, présenteront un comportement de liquéfaction, si l'essai est non drainé. En condition drainée, il y a un changement d'indice des vides et donc une modification de la surface d'effondrement. Ces surfaces d'effondrement représentent donc la limite de stabilité dans le seul cas où les conditions de drainage sont interdites.

Sladen suggère thypothèse suivante: la surface d'effondrement se modifie avec l'histoire de chargement comme la consolidation anisotrope, mais il n'existe pas de résultats expérimentaux étayant cette hypothèse.

Lade (1988) a donné un critère de stabilité pour le sable lâche en condition non drainée appelé « ligne d'instabilité ’ pour des échantillons consolidés isotropiquement. Ce critère est une droite, dans le plan $p^{\prime}-q$, passant par l'origine des axes et par les pics du déviateur du chemin des contraintes non drainés, pour différentes pressions de consolidation et pour le même indice des vides initial. La pente de la ligne d'instabilité augmente avec la diminution de l'indice des vides, c'est-à-dire avec l'augmentation de la densité relative des échantillons. Au-dessus d'une certaine densité relative, le phénomène de liquéfaction ne se vérifie plus.

Kramer et Seed (1988) confirment et montrent l'influence très défavorable de la présence d'un déviateur initial de contrainte sur la résistance à la liquéfaction statique du sable lâche consolidé isotropiquement. Si le niveau de déviateur initial augmente, la résistance à la liquéfaction diminue considérablement. Les mêmes conclusions sont retrouvées par Canou et al. (1991) en étudiant le comportement du sable d'Hostun RF.

Di Prisco et al. (1995) montrent que la ligne d'instabilité de Lade dépend de l'histoire de chargement telle que la consolidation, isotrope ou anisotrope, la surconsolidation ou le niveau de déformation drainée avant la phase non drainée. La surconsolidation augmente l'inclinaison de la ligne d'instabilité quelle que soit la consolidation initiale. Une histoire de chargement triaxial drainé avec des déformations suffisamment grandes permet d'avoir un comportement non drainé avec écrouissage au lieu d'un comportement de liquéfaction. Lanier et al. (1993) montrent aussi que l'on peut liquéfier un échantillon de sable dense en condition non drainée s'il est soumis à une extension non drainée après une compression triaxiale drainée.

Presque la totalité de ces études sur sable très lâche a été faite à l'aide des essais de compression triaxiale. Les essais d'extension commencent seulement depuis quelques années à apparaître dans les études.

Ainsi, Vaid et al. (1990) ont montré que le comportement du sable d'Ottawa est différent en compression et en extension. Pour les auteurs, cette différence est due à l'anisotropie inhérente des échantillons préparés avec la méthode de déposition pluviale.

Georgiannou et al. (1990) ont présenté les résultats d'une étude expérimentale sur le comportement d'un mélange de sable et d'argile consolidé de manière anisotrope. Ils remarquent qu'une surconsolidation réduit le potentiel de liquéfaction en compression, tandis que la liquéfaction statique continue à se manifester en extension.

Hyodo et al. (1994) ont étudié le comportement triaxial non drainé en compression et en extension pour différents niveaux de déviateur initial sur des échantillons de sable Toyoura consolidés isotropiquement. Dans le cas d'extension, le niveau du déviateur initial positif de contrainte n'influence pas les chemins de contrainte ni les déformations.

Récemment, Matiotti et al. (1995) ont montré l'influence d'une consolidation anisotrope sur le comportement non drainé en extension, pour différentes valeurs d'anisotropie de consolidation et pour différentes valeurs de surconsolidation. En extension comme en compression la ligne d'instabilité de Lade est modifiée sensiblement par une consolidation anisotrope ou par une surconsolidation.

\section{2}

\section{Programme expérimental}

Le but de cette étude est de compléter et d'étendre les résultats dans le domaine concernant la liquéfaction statique du sable très lâche, à l'aide d'essais triaxiaux de compression et d'extension sur des échantillons consolidés isotropiquement et anisotropiquement. 
La démarche expérimentale a été menée, d'abord pour obtenir une description complète de la liquéfaction statique du sable d'Hostun RF très lâche aussi bien en compression qu'en extension, et confirmer l'existence de la ligne d'instabilité de Lade en extension. Ceci sur des échantillons consolidés isotropiquement.

Ensuite, l'influence de l'anisotropie de consolidation sur la ligne d'instabilité a été examinée pour ce qui concerne les essais de compression. La généralisation de ce concept d'instabilité dans le cas des essais d'extension a été abordée dans le même temps en analysant les effets de la consolidation anisotrope sur le comportement en compression et extension.

Tous les échantillons ont été fabriqués avec l'indice des vides initial e proche de l'unité et l'indice de densité $I_{d}$ inférieur à $15 \%$. Les compacités maximales et minimales $\left(e_{\operatorname{mix}}=1,041\right.$ et $\left.e_{\min }=0,648\right)$, ainsi que les caractéristiques physiques du sable d'Hostun RF utilisé dans cette étude sont celles présentées par Flavigny et al. (1990).
Pour tous les essais, le cisaillement non drainé a été piloté en déformations, avec une vitesse de déformation axiale constante de $0,21 \% / \mathrm{mn}$, jusqu'à $15 \%$ de déformation axiale en compression et - $10 \%$ en extension.

La première série d'essais triaxiaux non drainés en compression et en extension, a été réalisée sur des échantillons consolidés isotropiquement pour des valeurs de pression de consolidation comprises entre 50 et $300 \mathrm{kPa}$.

Les deuxième et troisième séries d'essais en compression et en extension ont été réalisées sur des échantillons consolidés anisotropiquement pour différentes valeurs du rapport de consolidation anisotrope $\mathrm{K}=\sigma_{3}^{\prime} / \sigma_{1}^{\prime}$ et pour des valeurs de pression moyenne de consolidation p' comprises entre 100 et $400 \mathrm{kPa}$.

Une présentation d'ensemble des essais réalisés avec leurs conditions expérimentales est donnée dans le tableau I. Dans les noms des essais, le symbole "In signifie une consolidation isotrope, «KA , « KB $)$, " $\mathrm{KC}$ » une consolidation anisotrope avec les valeurs du rapport $K$, dans l'ordre, $0,66,0,50$ et 0,35 , «C $)$ et $\alpha E n$ le

TABLEAUI L'ensemble des essais non drainés sur sable Hostun RF très lấche.

\begin{tabular}{|c|c|c|c|c|c|c|c|}
\hline Essais & K & $e_{0}$ & $e_{c}$ & $\begin{array}{l}\mathrm{P}^{\prime} \\
\mathrm{kPa}\end{array}$ & $\begin{array}{l}\mathrm{q}_{\text {ace }} \\
\mathrm{kPa}\end{array}$ & $\begin{array}{l}\mathrm{P}_{\text {ife }}^{\prime} \\
\mathrm{KPa}\end{array}$ & $\varepsilon_{i p h}$ \\
\hline I-C1 & 1 & 1,044 & 0,929 & 50 & 20,02 & 35,36 & 0,208 \\
\hline $\mathrm{I}-\mathrm{C} 2$ & 1 & 0,994 & 0,883 & 100 & 42,79 & 71,55 & 0,297 \\
\hline 1-C3 & 1 & 0,985 & 0.875 & 200 & 94,23 & 137,90 & 0,422 \\
\hline I-C4 & 1 & 0,985 & 0,854 & 300 & 127.40 & 205,80 & 0,442 \\
\hline$[-E]$ & 1 & 1,058 & 0,948 & 53 & $-16,91$ & 44,05 & $-0,120$ \\
\hline I-E2 & 1 & 1,024 & 0,910 & 100 & $-32,42$ & 80,75 & $-0,170$ \\
\hline 1-E3 & 1 & 1,025 & 0,861 & 200 & $-56,89$ & 149,80 & $-0,290$ \\
\hline I-E4 & 1 & 1,048 & 0,899 & 300 & $-86,92$ & 210,00 & $-0,520$ \\
\hline $\mathrm{KA}-\mathrm{C} 1$ & 0,66 & 1,016 & 0,933 & 100 & 53,37 & 76,36 & 0,190 \\
\hline $\mathrm{KA} \cdot \mathrm{C} 2$ & 0,66 & 0,979 & 0,844 & 200 & 107,90 & 171,10 & 0,140 \\
\hline $\mathrm{KA}-\mathrm{C} 3$ & 0,66 & 1.020 & 0,849 & 300 & 161,90 & 271,90 & 0,095 \\
\hline $\mathrm{KA}-\mathrm{C} 4$ & 0,66 & 1,034 & 0,831 & 400 & 215,80 & 350,80 & 0,113 \\
\hline KA-E1 & 0,66 & 1,014 & 0,888 & 100 & $-21,90$ & 53,55 & $-0,430$ \\
\hline $\mathrm{KA}-\mathrm{E} 2$ & 0,66 & 1.024 & 0,885 & 200 & -55.80 & 109,40 & $-0,790$ \\
\hline $\mathrm{KA}-\mathrm{E} 4$ & 0,66 & 1,029 & 0,834 & 400 & $-86,40$ & 204,30 & $-1,040$ \\
\hline $\mathrm{KB}-\mathrm{C} 1$ & 0,50 & 1,031 & 0,889 & 100 & 77,20 & 95,50 & 0,030 \\
\hline $\mathrm{KB}-\mathrm{C} 2$ & 0.50 & 1,063 & 0,906 & 200 & 152,50 & 193,20 & 0,025 \\
\hline $\mathrm{KB}-\mathrm{C} 3$ & 0,50 & 1,021 & 0,842 & 300 & 234,90 & 290,50 & 0,050 \\
\hline $\mathrm{KB}-\mathrm{C} 4$ & 0,50 & 1,034 & 0,870 & 400 & 328,60 & 395,70 & 0,036 \\
\hline KB-E1 & 0,50 & 1,009 & 0,849 & 100 & $-14,20$ & 40,88 & $-0,604$ \\
\hline $\mathrm{KB}-\mathrm{E} 2$ & 0,50 & 1,040 & 0,851 & 200 & $-40,80$ & 90,90 & $-0,784$ \\
\hline $\mathrm{KB}-\mathrm{E} 3$ & 0.50 & 1,026 & 0,864 & 300 & $-50,54$ & 118,80 & $-0,859$ \\
\hline KB-E4 & 0.50 & 1,021 & 0,863 & 400 & $-95,20$ & 172,10 & $-1,410$ \\
\hline $\mathrm{KC}-\mathrm{C} 2$ & 0,35 & 1,016 & 0,843 & 200 & 232,20 & 196,20 & 0,035 \\
\hline
\end{tabular}


mode de chargement en compression, respectivement en extension. Les valeurs e représentent les indices des vides à la fin de consolidation isotrope ou anisotrope.

\section{3}

\section{Dispositif expérimental}

Le dispositif expérimental du laboratoire Géomatériaux est composé d'une cellule triaxiale pour des échantillons de $70 \mathrm{~mm}$ de diamètre et d'élancement 1,0, d'une presse électromécanique de type Farnell et d'un système de contrôle et d'acquisition de données par ordinateur.

Les grandeurs physiques de sorties sont mesurées à laide des capteurs, en nombre de deux pour la force axiale (un à l'intérieur de la cellule et l'autre à l'extérieur), de deux pour les pressions interstitielles et de confinement, de deux capteurs de déplacement de type LVDT pour la déformation axiale externe et interne (posé sur la tête supérieure de l'échantillon) et d'un capteur pour la variation de volume.

Un régulateur de pression équipé d'un moteur électrique permet de contrôler par ordinateur la pression de la cellule et de suivre à l'aide du capteur de force interne, n'importe quel chemin dans le plan triaxial des contraintes (Doanh et al., 1985).

Le système des têtes rotulées inférieure et supérieure avec des embases élargies de téflon, ainsi que le système d'accrochage permettant de réaliser des essais en extension, sont décrits en détail dans Robinet (1980) et Mohkam (1983). Une seule modification, face à de faibles valeurs de la déformation axiale au pic du déviateur non drainé du sable très lâche, a été faite en supprimant les deux couches habituelles de latex et de graisse de silicone entre les têtes et l'échantillon.

\section{4}

\section{Procédure expérimentale}

\section{1}

\section{Fabrication des échantillons}

Pour obtenir des échantillons avec des structures suffisamment lâches et contractantes pendant le cisaillement, la méthode de fabrication employée a été inspirée principalement par les travaux de Bjerrum et al., (1961), Ladd (1978).

Le sable avec une teneur en eau de $1 \%$ est déposé délicatement en quatre couches, à l'aide d'une petite cuillère et à la hauteur de chute nulle dans un moule cylindrique. Une membrane en néoprène de $0,3 \mathrm{~mm}$ d'épaisseur est plaquée préalablement à l'intérieur de ce moule. Les couches de sable de masses déterminées sont mises en place puis compactées aux différentes hauteurs de façon à prendre en compte le tassement supplémentaire imposé par compactages successifs. La densification jusqu'à la hauteur désirée est faite à l'aide d'un petit dameur de $3,5 \mathrm{~cm}$ de diamètre.

Lorsque le moule est rempli, la tête supérieure est fixée sur l'échantillon, la membrane est rabattue et fixée par un joint torique. Après avoir appliqué un vide de $10 \mathrm{kPa}$ à l'intérieur de l'échantillon pour assurer sa stabilité, on effectue le démoulage. Un tassement vertical a été observé et mesuré systématiquement après chaque démoulage.
Les dimensions initiales de l'échantillon (la hauteur et le diamètre moyen) ont été soigneusement prises et enregistrées. L'indice des vides initial $e_{0}$ a été calculé avec ces mesures.

\section{2}

\section{Saturation et consolidation}

Lorsque la cellule triaxiale est montée et remplie d'eau, une pression isotrope de $20 \mathrm{kPa}$ est appliquée en deux étapes. D'abord $10 \mathrm{kPa}$ et ensuite progressivement par des incréments unitaires, tout en diminuant jusqu'à zéro le vide à l'intérieur de l'échantillon.

Après avoir fait circulé du gaz carbonique $\mathrm{CO}_{2}$ sous un faible gradient de pression (d'environ 2 à $3 \mathrm{kPa}$ ) pendant 30 minutes, on fait passer de l'eau déminéralisée et désaérée pendant quelques heures à travers l'échantillon, jusqu'à ce que la quantité totale d'eau transitée soit au moins égale à deux fois le volume initial de l'échantillon.

Les déformations axiales pendant toutes les phases, y compris la phase de saturation, sont enregistrées automatiquement à l'aide d'un capteur de déplacement axial de type LVDT posé sur la tête supérieure de l'échantillon. Ces mesures synthétisées avec des résultats obtenus dans des essais de consolidation isotrope montrant que la déformation volumique est environ 2,7 fois la déformation axiale, ont permis d'estimer la variation de l'indice des vides pendant la phase de saturation.

Après cette percolation d'eau désaérée, en gardant toujours la contrainte effective de $20 \mathrm{kPa}$, on augmente manuellement en même temps la contre-pression et la pression de la cellule. La contre-pression utilisée est de $200 \mathrm{kPa}$, ce qui est suffisamment élevée pour dissoudre le gaz carbonique et obtenir des coefficients de Skempton supérieurs à 0,98 .

Les essais consolidés isotropiquement de la première séries sont amenés au point de départ isotrope en ajustant manuellement la contrainte de cellule. Lorsque la consolidation est complète, les têtes rotulées sont raccrochées avant le commencement des essais. Les variations de volume pendant la consolidation sont enregistrées et les valeurs de l'indice des vides corrigées.

Pour les deux dernières séries, la consolidation anisotrope est contrôlée par l'ordinateur en suivant un chemin en escalier d'amplitude $1,5 \mathrm{kPa}$ dans le plan p-q à partir d'un point décalé de $20 \mathrm{kPa}$ sur l'axe de pression moyenne effective jusqu'au point final de la consolidation. Techniquement, il n'est pas possible de réaliser des chemins de consolidation à partir de l'origine des axes.

Dans le tableau I sont présentées les valeurs corrigés de l'indice des vides après consolidation isotrope ou anisotrope e. Il n'y a pas de correction de membrane pour tous les essais présentés.

\section{5}

\section{Résultats expérimentaux}

\section{1}

\section{Consolidation isotrope}

Le but de la première série d'essais a été la vérification du comportement des échantillons consolidés isotropiquement (Fig. 1). 
Tous ces essais, aussi bien en compression qu'en extension, à valeurs d'indice des vides choisies, ont montré un comportement contractant pendant la phase de cisaillement non drainé.

L'allure du déviateur des contraintes (Fig. 1a) est caractérisée par un pic (maximum pour la compression et minimum pour l'extension) atteint pour une faible valeur de la déformation axiale, entre $\pm 0,5 \%$ (Fig. 2a). Le pic est suivi par une chute importante jusqu'à un état résiduel dans lequel l'échantillon se déforme à déviateur constant. Le déviateur montre une légère augmentation à partir de $8 \%$ de déformation axiale en compression et $-6 \%$ en extension.

Une augmentation de la valeur du pic de résistance et de la déformation axiale correspondante a été observée avec l'augmentation de la pression de confinement. Dans la figure 2a les pics du déviateur (marqués par des flèches) s'ordonnent de manière croissante en fonction de la pression de confinement. Autour de l'origine des axes, il y a une continuité entre les courbes en compression et en extension, c'est-à-dire des modules initiaux pour les différentes pressions de consolidation.

Les surpressions interstitielles (Fig. 1b) augmentent progressivement avec la déformation axiale, jusqu'à un

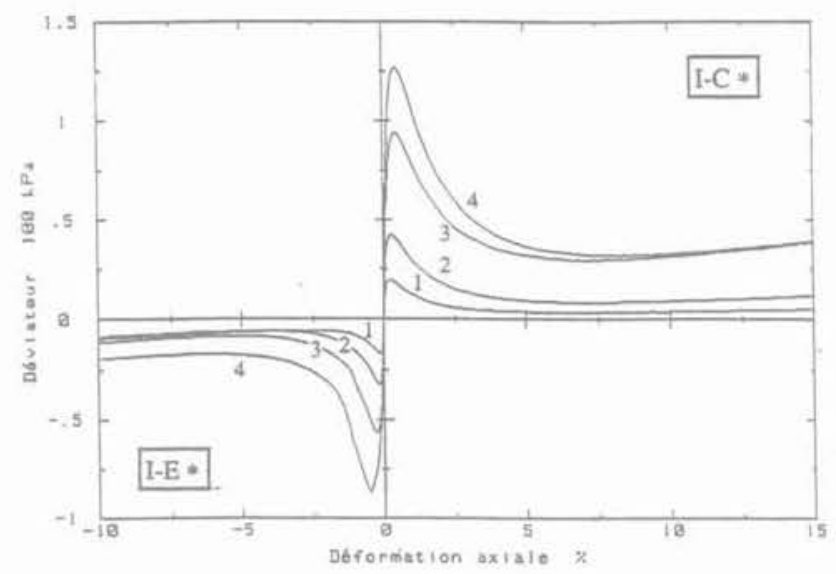

FIG. 1a Relation déviateur-déformation axiale essais isotropes.

Relationship deviator stress-axial strain for isotropic specimens.

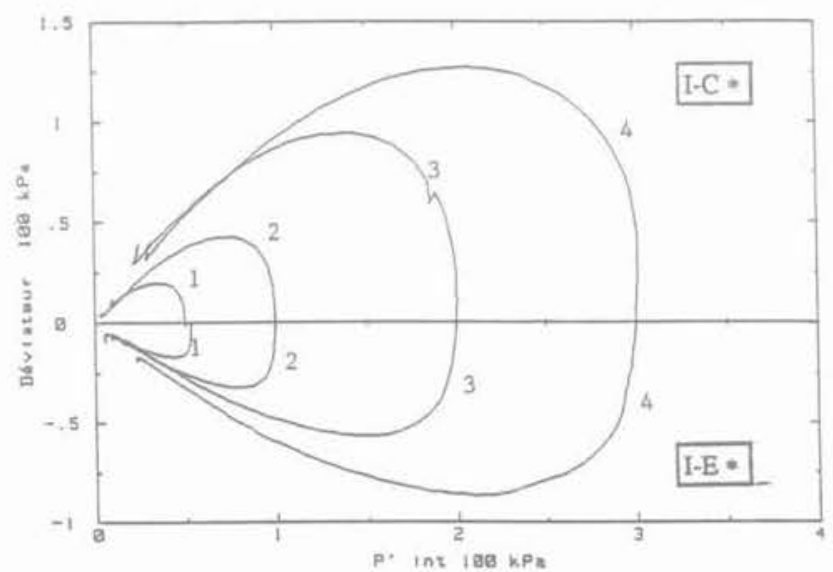

FIG ic Chemins de contrainte pour les essais isotropes en compression et extension. Stress paths for isotropic specimens in compression and extension tests. niveau constant atteint pour des valeurs de déformation axiale supérieures à $5 \%$ en compression et $4 \%$ en extension. En extension, la surpression interstitielle atteint $90 \%$ de la contrainte initiale effective de consolidation, tandis qu'en compression cette surpression monte jusqu'à $94 \%$ pour les essais I-C2, C3, C4 et $96 \%$ pour l'essai I-C1 consolidé à $50 \mathrm{kPa}$. La liquéfaction statique, définie comme l'annulation totale de la pression moyenne effective $p$, n'est pas atteinte pour l'ensemble des essais. Par exemple, il reste $2 \mathrm{kPa}$ pour annuler complètement la contrainte radiale dans le cas de l'essai I-C1. La liquéfaction complète n'a été obtenue qu'en inversant le chemin de contrainte, c'est-à-dire en effectuant un grand cycle de décharge.

La figure $2 b$ montre en détail le début de la phase de cisaillement non drainé et met en évidence la phase initiale de dilatance des essais d'extension. Pour des déformations axiales inférieures à $0,2 \%$, la pression interstitielle diminue pour atteindre $5 \%$ de la contrainte isotrope initiale. Cette dilatation en extension augmente progressivement, de façon très régulière, avec l'augmentation de la pression de confinement. Ce phénomène s'inverse pour des déformations axiales de $0,2 \%$. Les échantillons se contractent de

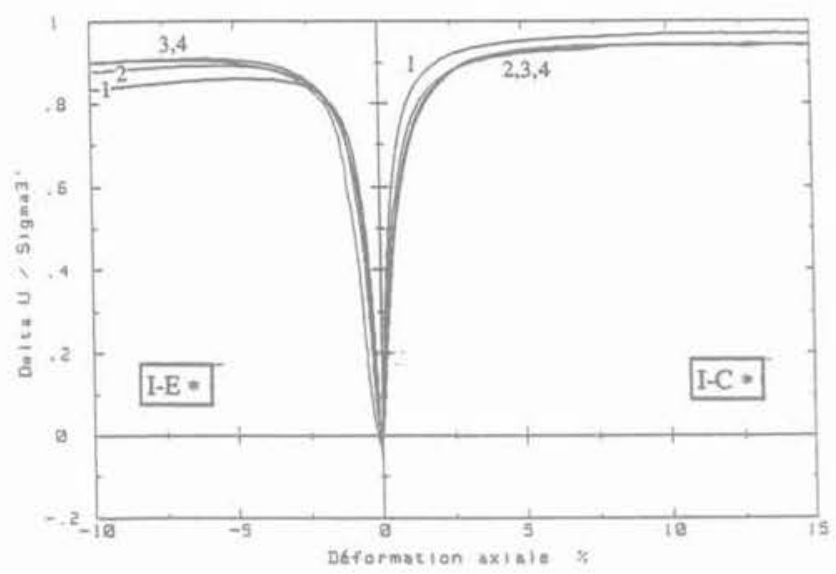

FG. 16 Courbes pression interstitielledéformation pour les essais isotropes. Pore pressure-strain curves for isotropic specimens.

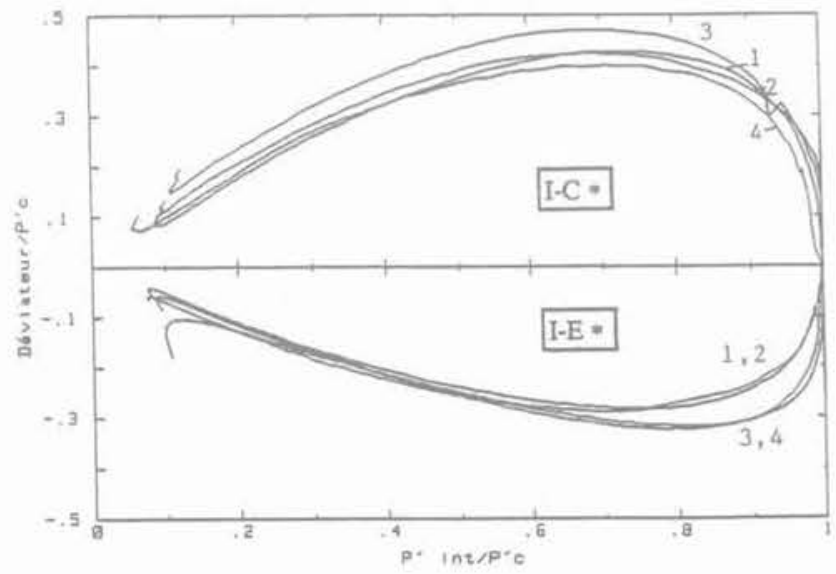

FG. 1d Normalisation des chemins de contrainte pour les essais isotropes.

Normalized stress paths for isotropic specimens. 
nouveau. Cette petite phase initiale de dilatance met en évidence la continuité du comportement au point de départ isotrope des essais. Puisque en compression la contractance forme un éventail de courbes de réponse augmentant proportionnellement avec la contrainte de consolidation, la continuité des réponses à l'origine impose une famille de courbes de dilatance, ce qui est démontré par les essais d'extension de la première série. Cette continuité de la réponse au point isotrope a été déjà remarquée par Mohkam (1983) sur des échantillons lâches d'un mélange de sables d'Hostun.

Les chemins de contrainte dans le plan q-p' sont présentés dans la figure 1c. A partir d'un point de l'axe isotrope, les deux chemins non drainés, en compression et en extension, forment une surface presque fermée vers l'origine des contraintes, légèrement asymétrique par rapport à l'axe des contraintes moyennes. La courbure, continue au passage de l'axe des contraintes moyennes, souligne une continuité déjà remarquée de la réponse du comportement au point isotrope. Une bonne homothétie entre les chemins est obtenue en normalisant les chemins de contraintes avec la pression moyenne effective initiale de consolidation (Fig. 1d).
Les états de contraintes correspondant au départ de la liquéfaction statique (pic de résistance) sont représentés dans la figure 3 en fonction des valeurs du déviateur et de la pression moyenne effective correspondante. Ainsi en compression comme en extension, les pics se trouvent alignés suivant des droites passant par l'origine. Ces droites, obtenues par régression linéaire (coefficients de régression très proches de 1), ont des pentes $\eta_{\text {ni }}$ de 0,64 en compression et $-0,41$ en extension. Autrement dit, $16,8^{\circ}$ pour compression et $12,7^{\circ}$ pour extension en terme de l'angle de frottement mobilisé au pic $\phi^{\prime}$ '

Konrad (1993) en présentant des essais réalisés à l'Institut de Mécanique de Grenoble pour le même sable et pour des échantillons isotropes avec des indices des vides $e=0,921 \pm 0,08$, trouve une valeur de $\eta_{\text {pic }}$ de 0,72 en compression.

Cette première série de huit essais sur des échantillons consolidés isotropiquement confirme les résultats du sable d'Hostun RF très lâche en compression, c'est-à-dire la liquéfaction statique, la ligne d'instabilité et le comportement contractant. En plus, elle montre l'existence de la ligne d'instabilité en extension, et valide le concept des lignes d'instabilité.

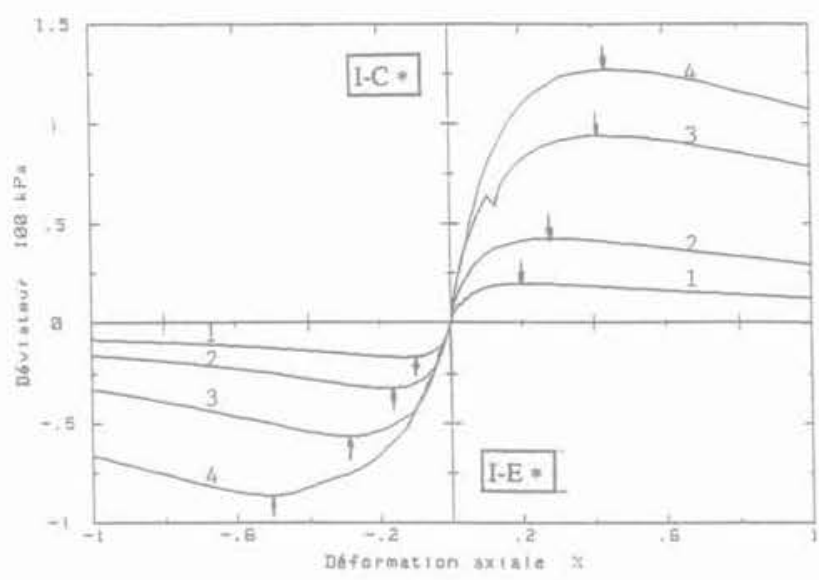

FiG. 2a Courbes déviateur-déformation axiale essais isotropes.

Deviator stress-axial strain curves for isotropic specimens.

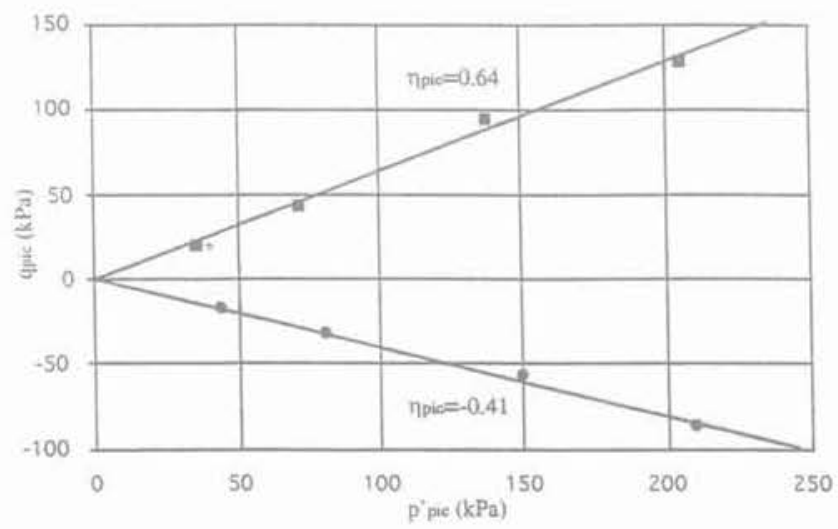

FIG.3 Droites d'instabilité en compression et extension pour les essais isotropes. Instability lines for isotropic specimens in compression and extension tests.

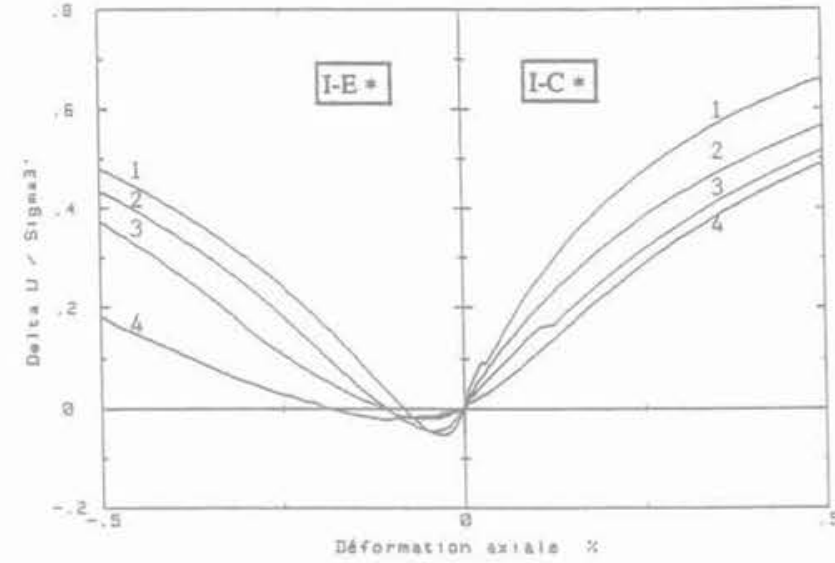

FIG. 26 Courbes pression interstitielledéformation pour les essais isotropes. Pore pressure-strain curves for isotropic specimens.

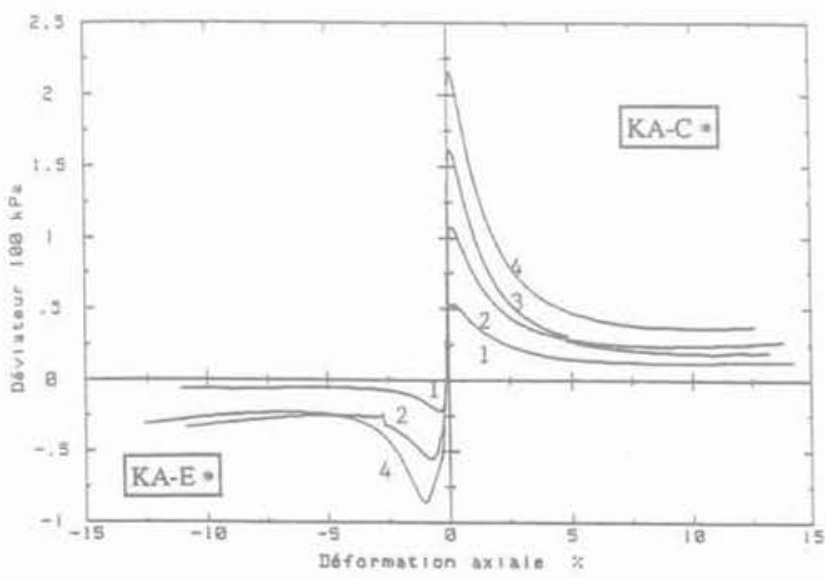

FiG. 4a Relations déviateur-déformation axiale essais anisotropes $\mathrm{K}=0,66$.

Relationship deviator stress-axial strain for anisotropic specimens with $\mathrm{k}=0.66$. 


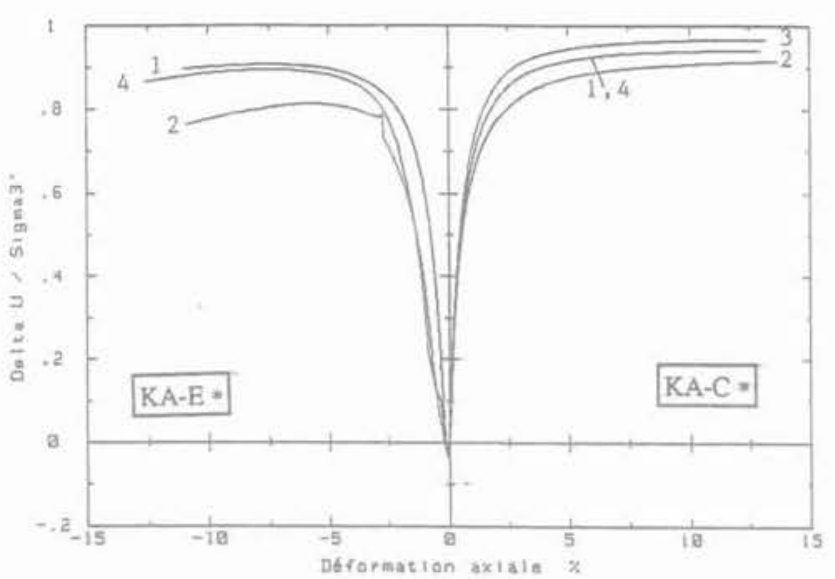

FG. 48 Courbes pression interstitielledéformation pour les essais anisotropes $\mathrm{K}=0,66$.

Pore pressure-strain curves for anisotropic specimens with $\mathrm{K}=0,66$,

\section{2}

\section{Consolidation anisotrope}

On définit le coefficient de consolidation anisotrope $\mathrm{K}$ comme le rapport entre la contrainte principale mineure $\sigma_{3}^{\prime}$ et la contrainte principale majeure $\sigma_{1}^{\prime}$. Après la série d'essais sur des échantillons consolidés isotropiquement, deux autres séries d'essais à consolidation anisotrope sont réalisées:

- une deuxième série d'essais (KA) pour un coefficient de consolidation $\mathrm{K}$ de 0,66 ;

- une troisième série (KB) pour un $\mathrm{K}$ de 0,50 correspondant à un niveau supérieur d'anisotropie.

Le premier rapport KA a été choisi car il donne, dans le plan $q-p^{\prime}$, une pente $\eta$ de consolidation de 0,44 , inférieure à la pente de la ligne d'instabilité identifiée par la première série d'essais $\left(\eta_{\text {puc }}=0,64\right)$. Le deuxième rapport $(K=0,50$ ou $\eta=0,75)$ situe la ligne de consolidation au-delà de la ligne d'instabilité et permet de tester la validité du concept des lignes d'instabilité sur des échantillons anisotropes.

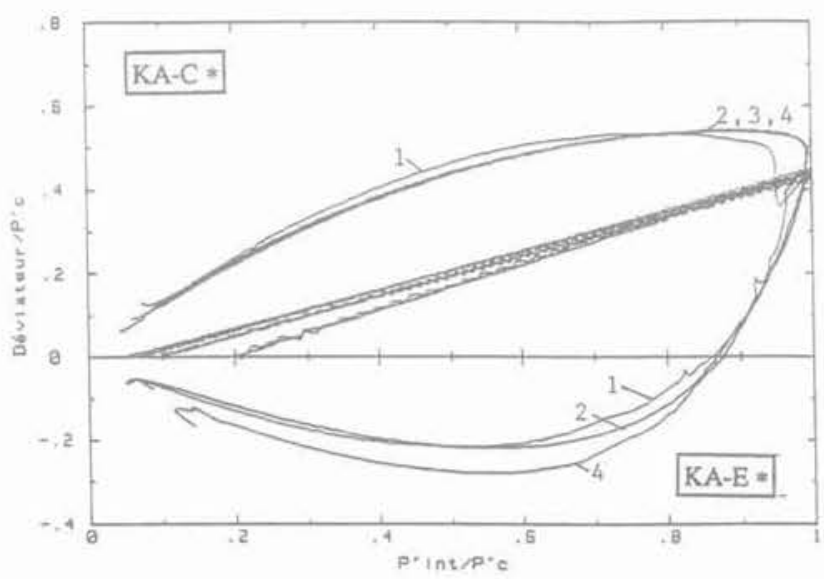

FiG. 4d Normalisation des chemins de contrainte pour les essais anisotropes $\mathrm{K}=0,66$. Normalized stress paths for anisotropic specimens with $K=0.66$.

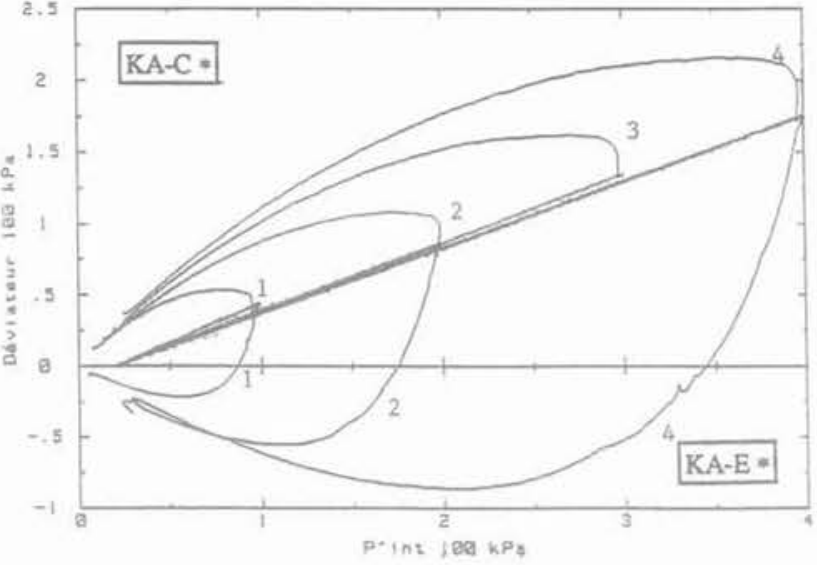

FG. AC Chemins de contrainte pour les essais anisotropes $\mathrm{K}=0,66$.

Stress paths for anisotropic specimens with $\mathrm{K}=0.66$.

Pour chaque série le comportement en compression et en extension pour différents niveaux de consolidation a été étudié. Pour la suite de cette étude, la pression moyenne effective à la fin du chemin de consolidation anisotrope est appelée pression de consolidation ( $\mathrm{p}^{\prime}$ )

\subsection{9: \\ Compression}

En ce qui concerne les essais en compression, cette étude retrouve des résultats proches de ceux présentés dans la littérature par Kramer et Seed (1988), Canou et al. (1991), pour des échantillons consolicés isotropiquement, mais suivis par l'application d'un déviateur de contrainte en condition drainée avant la phase non drainée. Les chemins de contraintes suivis par ces études ne sont pas de vrais chemins de consolidation anisotrope puisqu'ils atteignent le critère de rupture en augmentant le déviateur de contrainte, contrairement aux chemins de consolidation suivis par les deux dernières séries de cette étude.

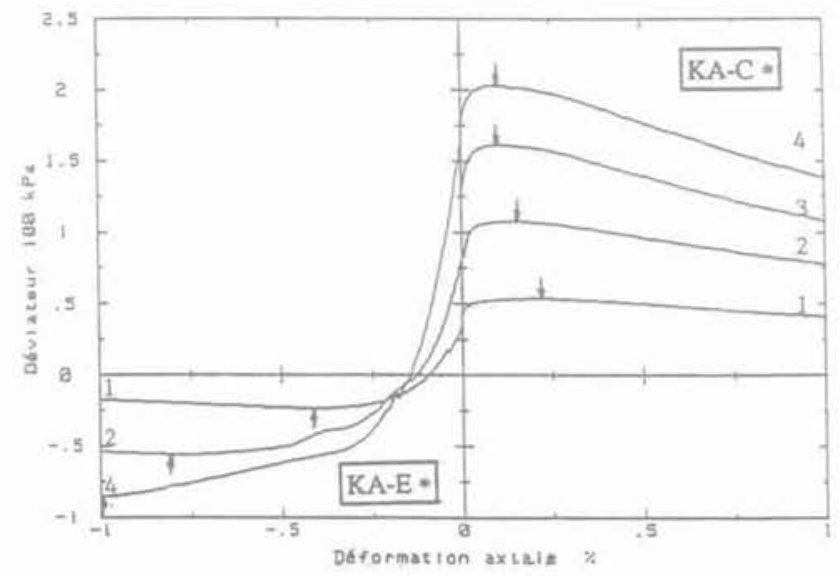

FG. 5a Courbes déviateur-déformation axiale essais anisotropes $\mathrm{K}=0,66$.

Deviator stress-axial strain curves for anisotropic specimens with $\mathrm{K}=0.66$. 


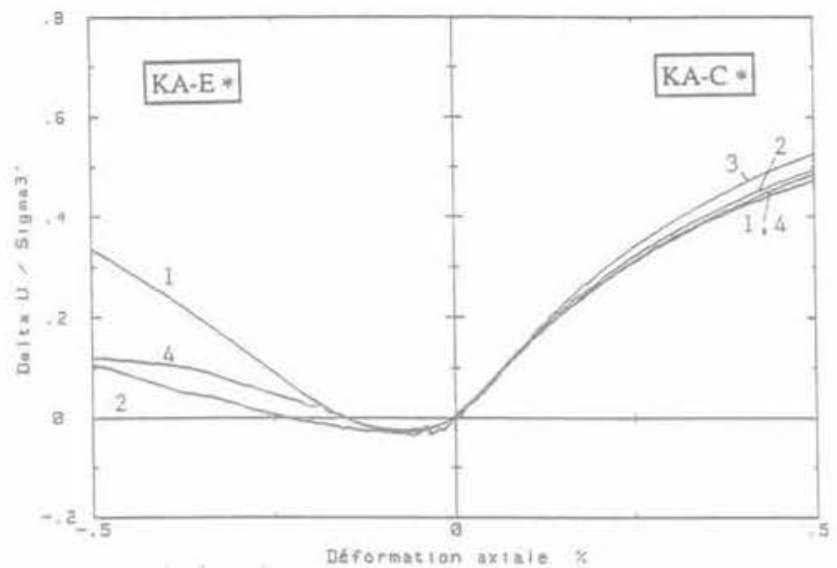

FIG. 5b Courbes pression interstitielledéformation pour les essais anisotropes $\mathrm{K}=0,66$.

Pore pressure-strain curves for anisotropic specimens with $K=0,66$.

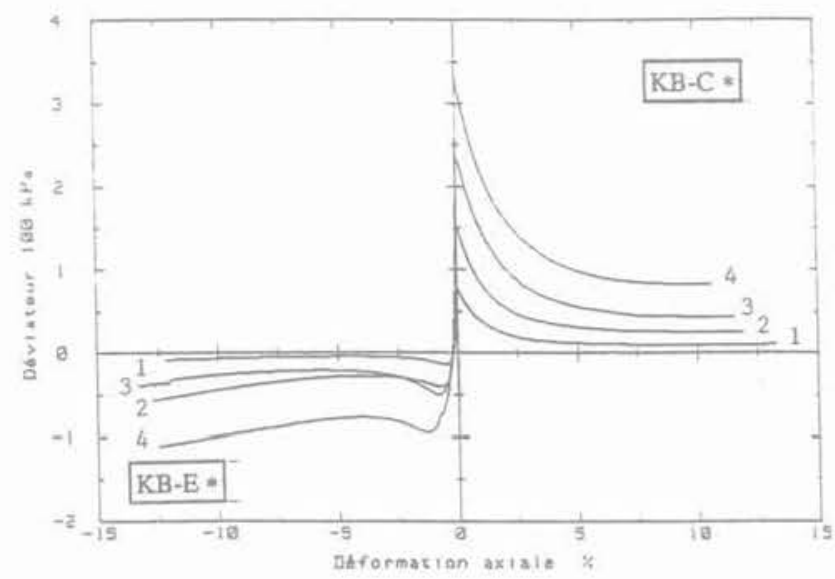

FG. $7 a$ Relations déviateur-cléformation axiale essais anisotropes $\mathrm{K}=0.50$.

Relationship deviator stress-axial strain for anisotropic specimens with $K=0.50$

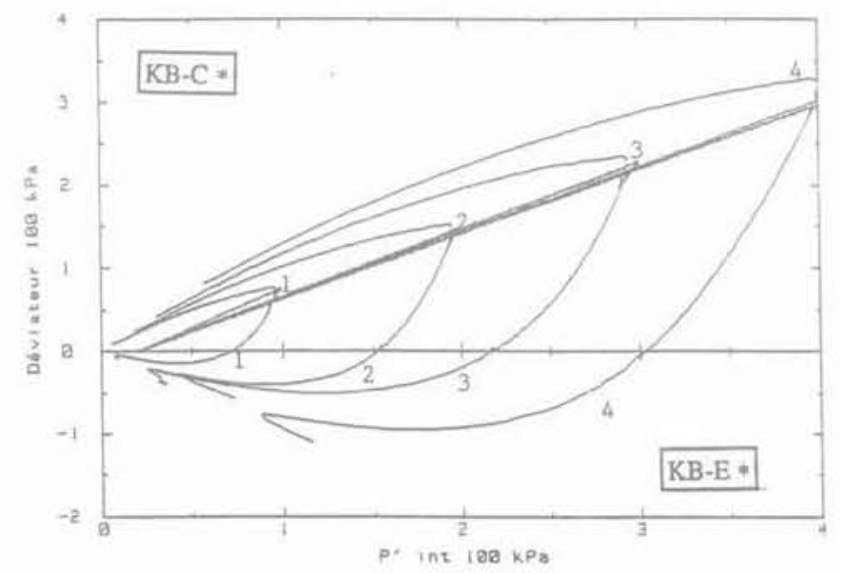

FIG. $7 c$ Chemins de contrainte pour les essais anisotropes $\mathrm{K}=0,50$ en compression et extension.

Stress paths for anisotropic specimens with $\mathrm{K}=0.50$ in compression and extension tests.

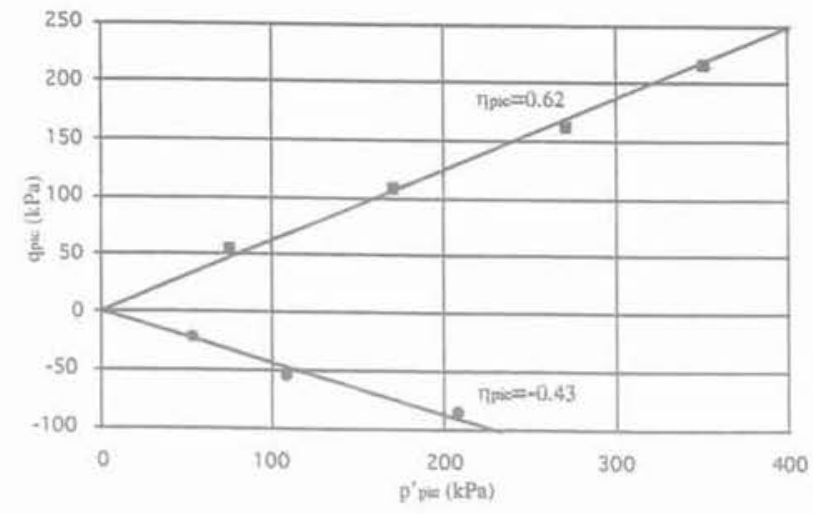

FG. 6 Droites d'instabilité en compression et extension pour les essais anisotropes $\mathrm{K}=0,66$,

Instability lines for anisotropic specimens with $\mathrm{K}=0.66$ in compression and extension tests.

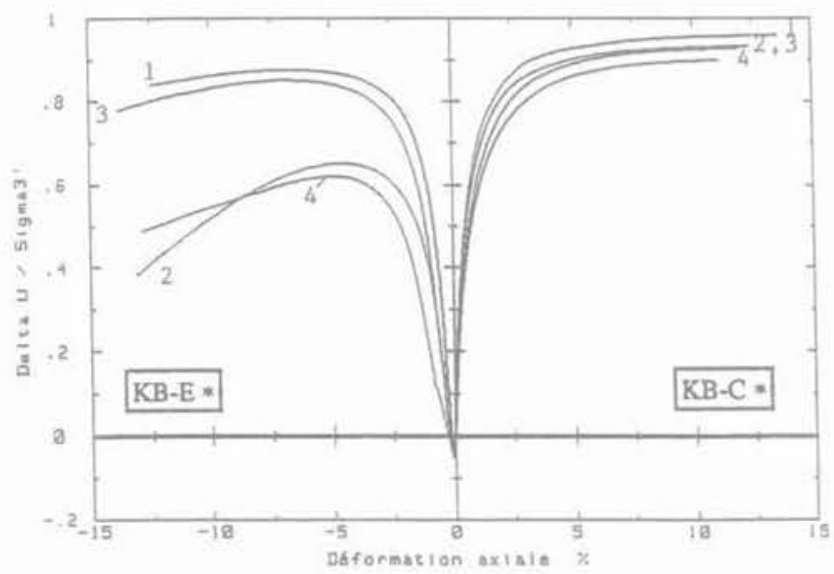

FG. 76 Courbes pression interstitielledéformation pour les essais anisotropes $\mathrm{K}=0,50$.

Pore pressure-strain curves for anisotropic specimens with $\mathrm{K}=0.50$.

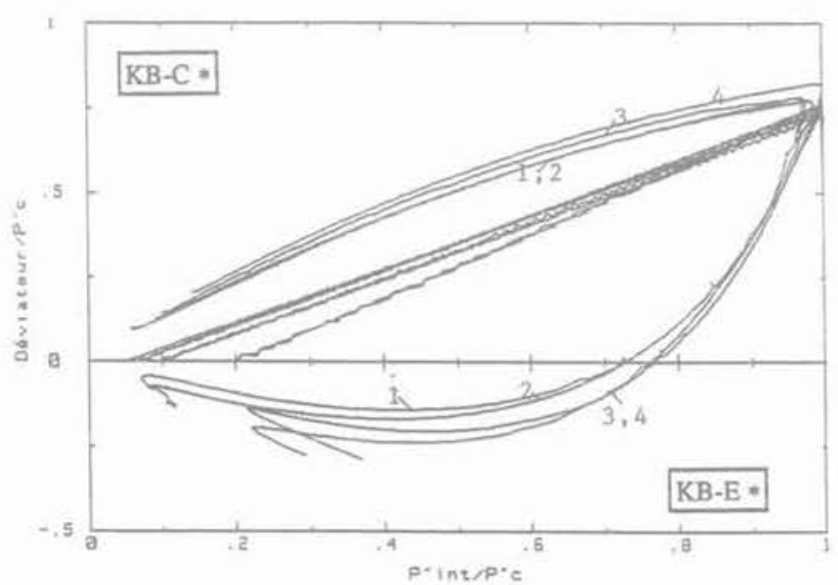

FG. Td Normalisation des chemins de contrainte pour les essais anisotropes $\mathrm{K}=0,50$. Normalized stress paths for anisotropic specimens with $\mathrm{K}=0.50$. 
Tous les essais, ceux de $\mathrm{K}=0,66$ ainsi que ceux de $\mathrm{K}$ $=0,50$, présentent, après la phase de consolidation, un pic du déviateur suivi par une chute très importante (Fig. $4 a$ et Fig. 7a).

La résistance mobilisée au pic de cisaillement non drainé, dans le cas de consolidation anisotrope, est définie par:

$$
\mathrm{q}_{\mathrm{u}}=\mathrm{q}_{\mathrm{pic}}-\mathrm{q}_{\mathrm{c}}
$$

avec: $q_{\text {pic }}=$ le déviateur au pic de résistance;

$\mathrm{q}_{\mathrm{r}}=$ le déviateur à la fin de la consolidation.

Cette résistance augmente avec la pression de consolidation $\mathrm{p}^{\prime}$, pour la même valeur de $\mathrm{K}$, et décroît avec la diminution de $\mathrm{K}$ pour la mème pression de consolidation. Dans la figure 10 sont représentées les valeurs de la résistance $q_{u}$ par rapport aux pressions de consolidation et aux valeurs de $K$, pour des essais anisotropes et isotropes.

Les valeurs de déformations axiales correspondantes aux pics sont toujours beaucoup plus petites que celles des essais avec consolisation isotrope (Fig. 5 a et Fig. 8a). Par contre, elles ne sont pas influencées par le niveau de la pression de consolidation (tableau I) puisqu'elles se trouvent dans une faible plage de $\varepsilon_{*}$ entre $0,1 \%$ et $0,2 \%$ pour $\mathrm{K}=0,66$ et $0,02 \%$ et $0,04 \%$ pour $\mathrm{K}=0,50$.

Les figures $4 \mathrm{~b}$ et $7 \mathrm{~b}$, montrent les courbes d'évolution des surpressions interstitielles normalisées par rapport à la contrainte effective radiale de consolidation en fonction de la déformation axiale. On retrouve les augmentations continues de la surpression jusqu'à des valeurs de $95 \%$ et de $90 \%$ de la pression effective radiale de consolidation, pour les essais avec $\mathrm{K}=0,66$, respectivement $K=0,50$. Ce sont des résultats analogues à ceux de la première série réalisées sur des échantillons consolidés isotropiquement. De même, la liquéfaction statique n'est pas atteinte entièrement selon la définition stricte de l'annulation de la pression moyenne effective. Par exemple, pour la plus petite pression de consolidation ( $\mathrm{p}_{\mathrm{f}}=100 \mathrm{kPa}$ ), il reste environ $0,50 \mathrm{kPa}$ avant d'atteindre la liquéfaction statique.

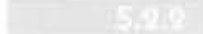

\section{Extension}

En ce qui concerne l'extension, le déviateur présente aussi un pic (minimum) suivi par une remontée jusqu'à une valeur résiduelle négative. Les déformations axiales aux pics sont comprises entre $-0,4 \%$ et $-1,5 \%$. Ces déformations aux pics augmentent, en valeurs absolues, avec les pressions de consolidation, comme celles de la série consolidée isotropiquement.

La valeur absolue de la résistance mobilisée au pic $\mathrm{q}_{\mathrm{u}}$ de cisaillement non drainé semble diminuer avec la reduction du rapport $\mathrm{K}$ et augmenter avec la pression de confinement pour le même rapport de consolidation.

Les valeurs stables de surpressions interstitielles sont atteintes pour des déformations axiales supérieures à $4 \%$ pour $\mathrm{K}=0,66$ et $3 \%$ pour $\mathrm{K}=0,50$ (Fig. $4 \mathrm{~b}$ et Fig. 7b). Pour les essais KB-E2 et KB-E4, la surpression atteinte n'est que $60 \%$ de la pression de confinement effective radiale et commence à diminuer à partir d'une déformation axiale de $6 \%$. Comme pour les essais d'extension isotrope, les figures $5 \mathrm{~b}$ et $8 \mathrm{~b}$ montrent une phase initiale dilatante qui génère une dimi- nution de la pression interstitielle avec des valeurs comprises entre $3 \%$ et $5 \%$ de la pression effective radiale de confinement.

Dans les figures $4 \mathrm{c}$ et $7 \mathrm{c}$ sont présentés les chemins de contraintes. Comme pour les essais avec consolidation isotrope, les chemins de contraintes, pour différentes pressions de consolidation, sont pratiquement homothétiques, en compression comme en extension, ainsi que le démontrent les courbes normalisées par rapport à la pression de consolidation (Fig. 4d et Fig. 7d).

Les points correspondant aux pics du déviateur et les valeurs des pressions moyennes effectives correspondantes, en compression comme en extension, sont indiqués dans les figures 6 et 9 . Ils sont très bien alignés, formant des droites passant par l'origine des axes. La régression linéaire donne de bons coefficients de corrélation ( $r=0,99$ en compression, $r=0,98$ en extension), ce qui confirme que le concept de la ligne d'instabilité peut être étendu au cas des essais en extension à consolidation anisotrope.

Comme pour le cas isotrope, dans le cas anisotrope, le niveau de contrainte au pic ou la résistance non drainée mobilisée est plus grande en compression qu'en extension.

Le tableau II regroupe les valeurs de l'angle de frottement et du rapport de contrainte au pic $\eta_{\text {pic }}$ pour tous les essais réalisés, ce qui permet d'analyser l'influence de l'anisotropie de consolidation sur cette ligne d'instabilité.

Pour les essais en compression avec consolidation $\mathrm{K}=0,66$ les niveaux de contrainte au pic sont presque identiques à ceux des essais avec consolidation isotrope, tandis que les essais $K=0,50$ obtiennent une valeur supérieure de $\eta$ d'environ 0,2 par rapport au cas isotrope, qui correspond à $5^{\circ}$ de différence entre les angles de frottement. Il semble donc que la pente de la ligne d'instabilité dépende du niveau de consolidation, par conséquent, ce concept d'instabilité n'a pas de valeur intrinsèque comme la rupture. Pour clarifier cet aspect, un essai supplémentaire de compression avec un coefficient de consolidation $\mathrm{K}=0,35$ et pour une pression de consolidation $\mathrm{p}_{c}^{\prime}$ de $200 \mathrm{kPa}$ est réalisé. La consolidation anisotrope de cet essai est très proche de la surface de rupture, et le point de départ de l'écrasement monotone en compression non drainé se situe nettement au-dessus de la droite d'instabilité d'échantillons isotropes.

TABLEAU II L'angle de frottement et le rapport de contrainte au pic de dêviateur.

\begin{tabular}{cccc}
$K$ & mocte & $\varphi_{\text {fic }}$ & $\eta_{\text {pic }}$ \\
\hline 1 & $C$ & $16^{\circ} 8$ & 0,64 \\
\hline 1 & $E$ & $12^{\circ} 7$ & $-0,41$ \\
\hline 0,66 & $C$ & $16^{\circ} 3$ & 0,62 \\
\hline 0,66 & E & $13^{\circ} 4$ & $-0,43$ \\
\hline 0,5 & $C$ & $21^{\circ} 3$ & 0,83 \\
\hline 0,5 & E & $15^{\circ} 9$ & $-0,50$ \\
\hline 0,35 & C & $29^{\circ} 1$ & 1,16 \\
\hline
\end{tabular}




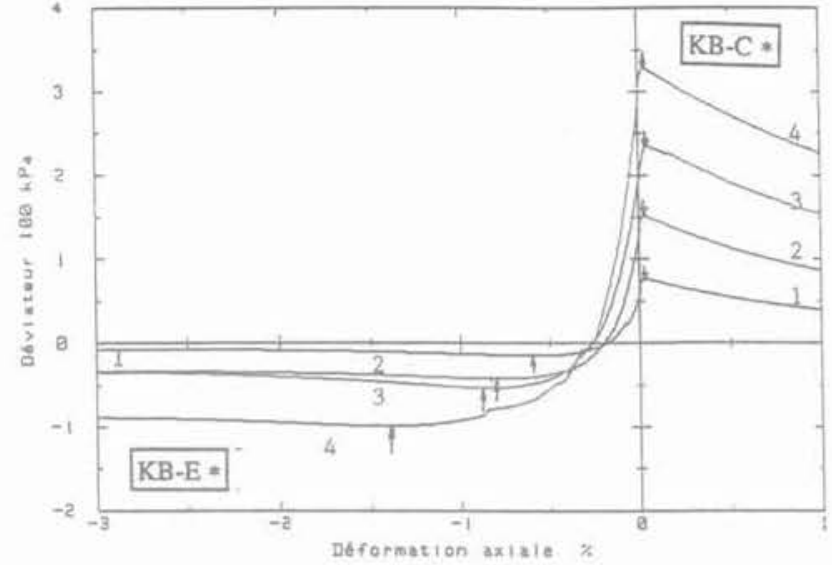

Fic Ba - Courbes déviateur-déformation axiale essais anisotropes $\mathrm{K}=0,50$.

Deviator stress-axial strain curves

for anisotropic specimens with $\mathrm{K}=0.50$.

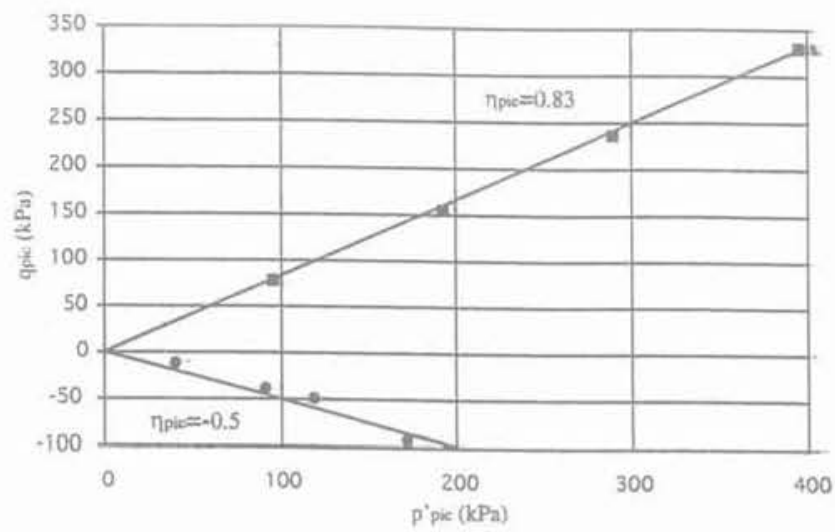

FG.9 Droites d'instabilité en compression et extension pour les essais anisotropes $\mathrm{K}=0,50$.

Instability lines for anisotropic specimens with $\mathrm{K}=0.50$ in compression and extension tests.

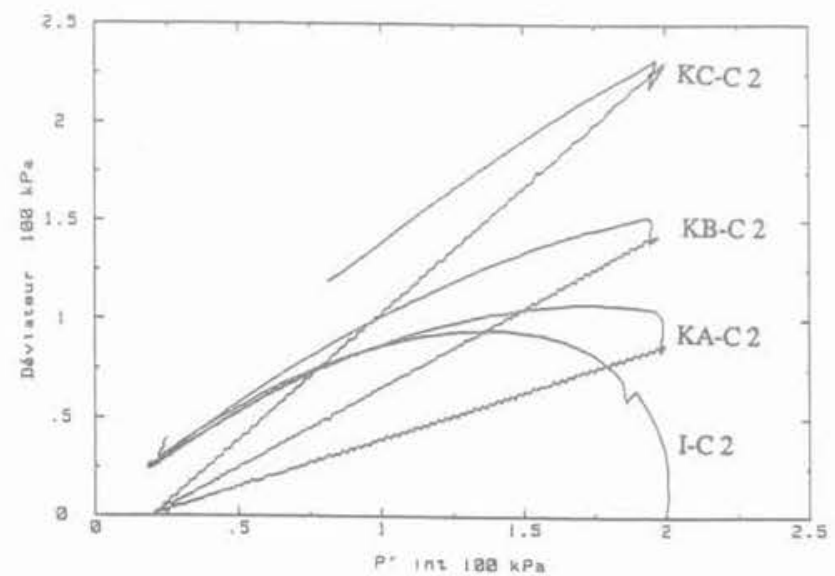

FG. 11 Chemins de contrainte pour les essais à $200 \mathrm{kPa}$ de pression de confinement. Stress paths for tests consolidated under $200 \mathrm{kPa}$.

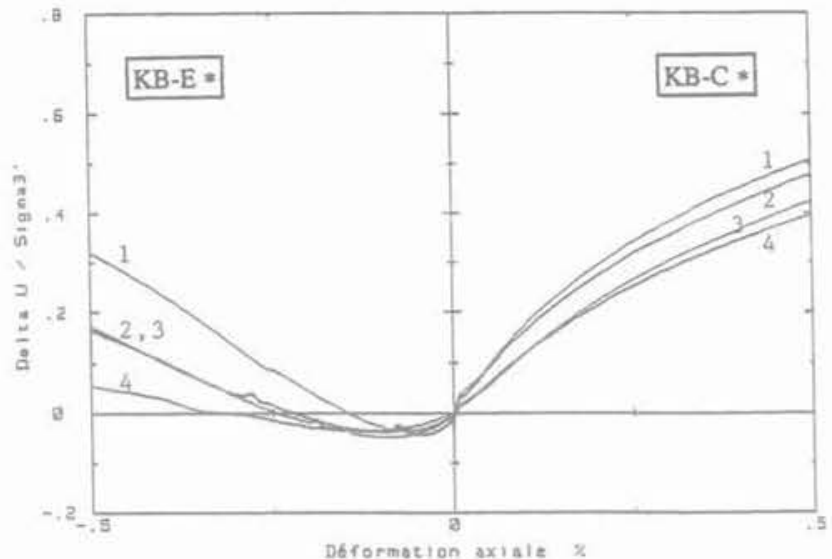

ศด. 86 Courbes pression interstitielledéformation pour les essais anisotropes $\mathrm{K}=0,50$.

Pore pressure-strain curves for anisotropic specimens with $\mathrm{K}=0.50$,

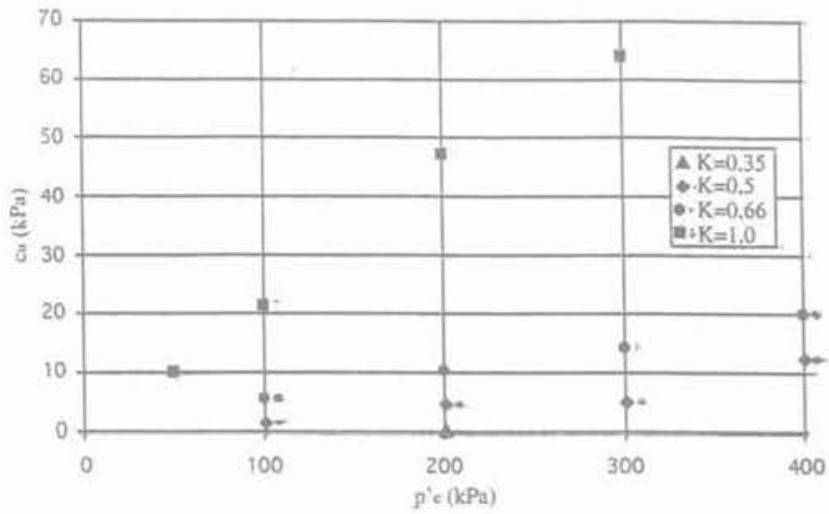

FIG 10 Relation entre la résistance mobilisée au pic de cisaillement non drainé et la pression de consolidation pour des essais anisotropes et isotropes. Plot of maximum undrained shear strength. versus consolidation pressure for anisotropic and isotropic tests.

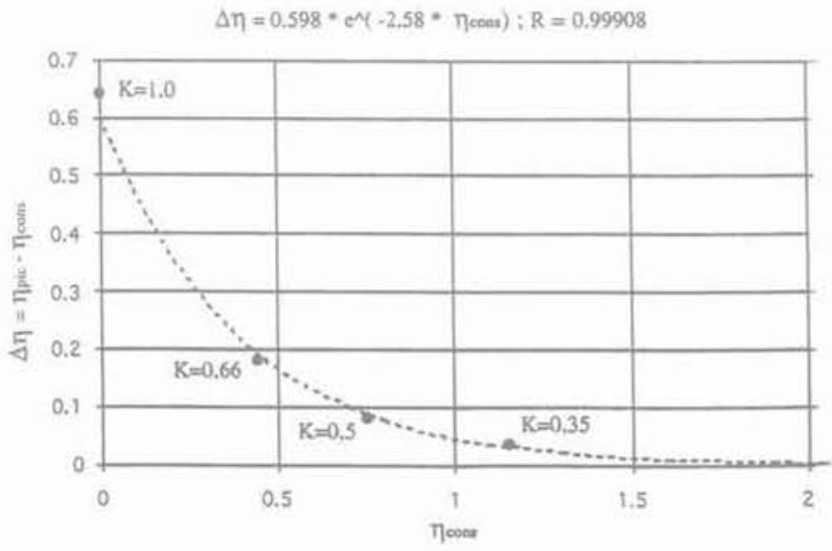

FIG.18 Normalisation des valeurs de contrainte au pic par rapport au niveau de contrainte de consolidation.

Stress peak level normalized by the stress consolidation level. 
L'ensemble des chemins de contrainte dans le plan $\mathrm{q}-\mathrm{p}^{\prime}$ pour les essais à $200 \mathrm{kPa}$ de pression de confinement est donné dans la figure 11. Le niveau de contrainte $\eta$ au pic de ce dernier essai est supérieur à celui de l'échantillon isotrope d'environ 0,5 , qui correspond à $12^{\circ}$ de différence entre les angles de frottement. Dans cette figure, l'essai KA-C2 à K =0,66 offre une résistance non drainée non négligeable de 10,2 kPa, ce qui est attendu selon le concept original d'instabilité, puisque cet essai a le point de départ non drainé audessous de la ligne d'instabilité. L'essai KB-C2 à $\mathrm{K}=0,50$ montre une résistance non drainée inattendue de $4,8 \mathrm{kPa}$, contrairement à la prévision établie avec la ligne d'instabilité des échantillons isotropes. Finalement, l'essai supplémentaire $\mathrm{KC}-\mathrm{C} 2$ à $\mathrm{K}=0,35$ confirme la dépendance du concept d'instabilité en fonction de la consolidation, puisqu'il existe une résistance non drainée, quoique faible, d'environ $0,50 \mathrm{kPa}$.

En extension, pour l'ensemble des essais présentés, la différence entre le cas de consolidation isotrope et anisotrope est un peu moins marquée. La différence de l'angle du frottement mobilisé au pic de résistance non drainée est d'environ $0,7^{\circ}$ entre les essais isotropes et ceux à $\mathrm{K}=0,66$; de $3^{\circ}$ entre les essais isotropes et ceux à $\mathrm{K}=0,50$. Cette différence indique une augmentation (en valeur absolue) plus progressive.

Une normalisation des valeurs de contrainte au pic par rapport au niveau de contrainte de consolidation, c'est-à-dire par rapport à l'anisotropie de consolidation, est présentée dans la figure 12. L'incrément de niveau de contrainte $\Delta \eta$ représenté par la différence entre les pentes au pic $\eta_{\text {pic }}$ et à la fin de la consolidation $\eta_{\text {cons' }}$, suit une diminution continue avec l'augmentation de la pente de consolidation, modélisée selon une courbe exponentielle décroissante. Les quatre expériences de la figure 11 permettent d'obtenir les coefficients numériques de cette courbe.

$$
\Delta \eta=0,598 * \exp \left(-2,58 * \eta_{\text {cons }}\right)
$$

L'expérience supplémentaire à $\mathrm{K}=0,35$ s'insère convenablement dans la continuité des trois essais précédents.

Au niveau correspondant à la rupture drainée, $\eta_{\text {con. }}$ estimée à 1,4 , ce modèle fournit une très petite résistance non drainée, environ $0,3 \mathrm{kPa}$ pour $200 \mathrm{kPa}$ de $\mathrm{p}^{\prime}$. Mais, au laboratoire, il est pratiquement impossible de suivre un chemin de consolidation situé très près de la surface de rupture, pour vérifier cette prévision.

Ces expériences montrent qu'en augmentant le niveau de l'anisotropie de consolidation, il y a toujours un pic du déviateur en condition de cisaillement non drainé, même si la valeur du pic diminue de plus en plus. Autrement dit, elles mettent en évidence l'influence du chemin de consolidation dans le concept des lignes d'instabilité.

\section{6}

\section{Conclusions}

Dans cet article une étude expérimentale sur le comportement non drainé de sable d'Hostun RF très lâche sous consolidation isotrope et anisotrope, en extension et en compression a été présentée. Les conclusions suivantes peuvent être avancées :

1) La liquéfaction statique est un phénomène d'instabilité des sables très lâches. Ce phénomène existe aussi bien en compression qu'en extension, et quelle que soit thistoire de consolidation, isotrope ou anisotrope. Ce phénomène est caractérisé par un pic positif ou négatif du déviateur, suivi par une chute importante de résistance, jusqu'à un niveau auquel le déviateur se stabilise autour d'une valeur résiduelle. La deuxième caractéristique de ce phénomène est l'annulation de la pression moyenne effective en écrasement monotone non drainé.

2) Le concept de ligne d'instabilité de Lade est corroboré en extension sur les échantillons consolidés isotropiquement. Ce concept peut être étendu sur les échantillons anisotropes.

3) La ligne d'instabilité est fortement influencée par I'histoire de consolidation. Une forte anisotropie de consolidation a pour effet de relever sensiblement la pente de cette ligne d'instabilité.

4) Quelle que soit l'histoire de consolidation, il existe une résistance non drainée à mobiliser en compression comme en extension. Le niveau de contrainte au pic augmente continuellement avec l'augmentation du niveau d'anisotropie de consolidation. Mais l'incrément du niveau de contrainte au pic, par rapport au niveau de contrainte de consolidation, diminue.

\section{Remerciements}

Les auteurs remercient la Communauté européenne pour le soutien financier par l'intermédiaire de son programme ALERT Géomatériaux. 
Bjerrum L., Kringstad S., Kummeneje D. The shear strenght of a fine sand. Proc. $5^{\text {th }}$ Int. Conf. Soil Mech. Found. Eng., Paris, vol. 1, 1961, p. 29-37.

Canou J., Thorel L., de Laure E. - Influence d'un déviateur de contrainte initial sur fes caractéristiques de liquéfaction statique du sable. Proc. X ECSMFE. Firenze, vol. 1, 1991, p. 49-52.

Casagrande A. - Liquefaction and cyclic deformation of sands: a critical review. Havard Soil Mechanics, Series n ${ }^{\circ} 88$, Havard University, Cambridge, MA, 1976, $51 \mathrm{p}$.

Castro G - Liquefaction of sands. Ph. D. Thesis, Havard Soil Mechanics Series $n^{\circ} 81$, Havard University, Cambridge, MA, 1969

Di Prisco C., Matiotti R., Nova R. - Theoretical investigation of the undrained stability of shallow submerged slopes. Geotechnique, vol. 45, $n^{\circ} 3$, sept. 1995 , p. $479-496$.

Doanh T., Mohkam M. - Influence of stress path on Hostun sand. Proc. Numeta 85, Swansea, 1985, p. 429-436.

Flavigny E., Desrues J., Palayer B. - Le Sable Hostun RF. Revue Française de Géotechnique, n53, 1990, p. 67-69.
Georgiannou V.N., Burland J.B., Hight D.W. - The undrained behaviour of clayey sands in triaxial compression and extension. Geotechnique, vol. $40, n^{2} 3$. 1990, p. 431-449.

Hyodo M., Tanimizu H., Noriyuki Y., Murata H. - Undrained cyclic and monotonic triaxial behaviour of saturated loose sand. Soils and Foundations. vol. 34, n० 1, 1994, p. 19-32.

Konrad J. - Undrained response of loosely compacted sands during monotonic and cyclic compression tests. Geotechnique. vol. $43, n^{\circ} 1,1993, p .69-89$.

Konrad 1 Flavigny E Megachou M Compontement non drainé du sable d'Hostun lâche. Revue Française de Géotechnique, $n^{2} 54,1991$, p. 53-63.

Kramer S.L., Seed H.B. - Initiation of soil licquefaction under static loading conditions. J. Geotech. Engrg., ASCE, vol. 114, $n^{\circ}$ GT4, 1988, p. 412-430.

Ladd R.S. - Preparing test specimens using undercompaction. Geotechnical Testing Journal, vol, $1, n^{\circ} 1,1978$, p. 16-23.

Lade P.V. Nelson R. B. Ito Y.M. - Instability of granular materials with nonassociated flow. J. Geotech. Engrg. ASCE. vol. 114, $n^{\circ}$ GT12, 1988, p. 2173-2191.
Lanier J., Di Prisco C., Nova R. - Etude expérimentale et analvse théorique de l'anisotropie induite du sable Hostun. Revue Française de Géotechnique, $n^{\circ} 57$. 1993, p. 59-74.

Matiotti R., Ibraim E., Doanh T. - Undrained behaviour of very loose RF sand in compression and extension tests. Numog V.. Davos, Suisse Ed. Pande and Pietruszczak, Rotterdam. Balkema, 1995, p. 119-124.

Mohkam M - Contribution à l'étude expérimentale et théorique du comportement des sables sous chargements cycliques. These DI. USTM, Grenoble. 1983

Poulos S.J. - The steady state of deformation. J. Geotech. Engrg., ASCE, vol. 107. $n^{\circ}$ GI5, 1981, p. 553-562.

Robinet J -C - Loi rhéologique en écriture incrémentale. Application aux sols et au béton. Thèse DE, USTM Grenoble, 1980.

Sladen IA DHollander P D KrahnI. The liquefaction of sands, a collapse surface approach. Can. Geotech I., vol.22. $n^{\circ} 4,1985$, p. $564-578$.

Vaid Y.P., Chung E.K.F., Kuerbis R.H. Stress path and steady state. Can. Geotech. J., vol. $27, n^{\circ} 1,1990$, p. 1-7. 